\title{
Radiation Effect on MHD Stagnation-point Flow of a Nanofluid over an Exponentially Stretching Sheet in the Presence of Chemical Reaction
}

\author{
G. Narender ${ }^{1}$, G. Sreedhar Sarma ${ }^{2}$ and K. Govardhan ${ }^{3}$ \\ ${ }^{1}$ Asst. Professor, CVR College of Engineering / H \& S Department(Mathematics), Hyderabad, India. \\ Email: gnriimc@gmail.com \\ ${ }^{2}$ Assoc. Professor, CVR College of Engineering / H \& S Department(Mathematics), Hyderabad, India. \\ Email: sarma.sreedhar@gmail.com \\ ${ }^{3}$ Asst. Professor, GITAM University, Department of Mathematics, Hyderabad, India. \\ Email: govardhan_kmtm@yahoo.co.in
}

\begin{abstract}
The effect of chemical reaction parameter on magnetohydrodynamic boundary layer flow of nanofluid past an exponentially stretching sheet is presented. An appropriate set of similarity transformations are used to transform the governing partial differential equations (PDEs) into a system of nonlinear ordinary differential equations (ODEs). The resulting system of ODEs is solved numerically by using the $4^{\text {th }}$ order Adam's - Moultan method along with shooting technique. The numerical results are analyzed by graphs for different parameters which appear in the solution affecting the MHD mixed convection stagnation point.
\end{abstract}

Index Terms: Numerical solution, MHD, Radiation effect, stagnation-point flow, chemical reaction, Nanofluid.

\section{INTRODUCTION}

The study of heat and mass transfer on fluids with chemical reaction effect past an exponentially stretching surface have important role in chemical engineering, nuclear reactors, solar collectors, drying processes, heat exchangers, geothermal and oil recovery, building construction. Possible applications of this type of flow can be found in many industries. From technological point of view, MHD flow finds application in the field of stellar and planetary magneto-spheres, aeronautics, meteorology, solar physics, cosmic fluid dynamics, chemical engineering, electronics and induction flow merry, MHD generators, MHD accelerators and other centrifugal mechanics. Nanofluids have properties that make them potentially useful in many heat transfer applications. They exhibit enhanced thermal conductivity and convective heat transfer coefficient.

Choi [1] studied the enhancing thermal conductivity of fluid with nanoparticles. Buongiorno [2] presented a simple convective model to analyz transport in nanofluids implied that energy transfer by dispersion of nanoparticles was negligible. In the boundary layer, there may be a decrease in viscosity, which will lead to heat transfer enhancement. An excellent assessment of nanofluid physics and developments had been provided by Cheng [3]. Buongiorno and $\mathrm{Hu}$ [4] they analyzed the thermal conductivity in nano fluid and gave the comparison for the enhancement heat transfer. However, Gupta et al. [5] examined the heat and mass transfer for the boundary layer flow over a stretching sheet subject to suction and blowing. Emmanuel et al. [6] has examined heat and mass transfer in a visco elastic boundary layer flow over an exponentially stretching sheet. The impact of viscous dissipation, non-uniform heat source on heat transfer in a viscoelastic boundary layer flow over a stretching sheet was studied by Subhas et al. [7]. The effect of thermal radiation on the flow was examined by Sohail Nadeem et al. [8]. Al-odat et al. [9] obtained the results on the effect of magnetic field on an exponential temperature distribution on the linear sheet. Gopi Chand et al. [10] considered an unsteady stretching surface in a porous medium and explained the viscous dissipation and radiation effects on MHD flow over it. Ishak [11] worked on the radio ative magnetohydrodynamic boundary layer flow. Flow through a porous, bounded by a vertical surface in presence of hall current was explained by Sudhakar et al. [12]. B Bidin and $\mathrm{R}$ Nazar [13] presented the MHD boundary layer flow with radiation effect.

Our prime objective is, we first reproduce an analytical study of [14] and then extend the MHD stagnation point flow of nanofluid past an exponentially stretching sheet with convective boundary condition." According to our information, chemical reaction effects on MHD mixed convection stagnation point flow of nanofluid over an exponentially stretching surface" is not yet examined.

\section{MAThematical Formulation}

Consider the steady, two-dimensional boundary layer flow of a nanofluid over an exponentially stretching surface. The stretching and free stream velocities are assumed to be $u_{w}(x)=a e^{(x / l)}$ and $u_{\infty}(x)=b e^{(x / l)}$ where $a$ and $b$ are constants. In addition, a magnetic field $B(x)=B_{0} e^{(x / 2 l)}$ is applied to the plate as shown in figure 1. Temperature and concentration are denoted by $\mathrm{T}$ and $C$ with suffix $w$ and $\infty$ for 
stretching surface and free stream respectively take forms $T_{w}(x), C_{w}(x), T_{\infty}$ and $C_{\infty}$.

The governing equations of conservation of momentum, energy, mass and nanoparticle fraction, under the boundary layer approximation, are as follows:

$$
\frac{\partial u}{\partial x}+\frac{\partial v}{\partial y}=0
$$

$u \frac{\partial \mathrm{u}}{\partial \mathrm{x}}+\mathrm{v} \frac{\partial \mathrm{v}}{\partial \mathrm{y}}=v \nabla^{2} u+U_{\infty} \frac{\partial U_{\infty}}{\partial x}+\frac{\sigma B_{0}^{2}}{\rho_{f}}\left(U_{\infty}-u\right)$

$u \frac{\partial \mathrm{T}}{\partial \mathrm{x}}+\mathrm{v} \frac{\partial \mathrm{T}}{\partial \mathrm{y}}=\alpha \nabla^{2} T-\frac{1}{\rho c_{p}} \frac{\partial q_{r}}{\partial y}+\frac{v}{\rho c_{f}}\left(\frac{\partial u}{\partial y}\right)^{2}+\tau\left(D_{B} \nabla \varphi \cdot \nabla T+\right.$

$\left.\frac{D_{T}}{T_{\infty}} \nabla T . \nabla T\right)$

$u \frac{\partial \varphi}{\partial \mathrm{x}}+\mathrm{v} \frac{\partial \varphi}{\partial \mathrm{y}}=\alpha \nabla^{2} T+\frac{D_{T}}{T_{\infty}} \nabla^{2} T-k_{0}\left(C-C_{\infty}\right)$

where $x$ is the coordinate axis along the continuous surface in the direction of motion and $y$ is the coordinate axis along the continuous surface in the direction perpendicular to the motion. The components of velocity along $x-$ and $y-$ axis are respectively $u$ and $v$. In the above equations, $\mu$ is the kinematic viscosity, $\rho_{f}$ is the density, $\sigma$ is an electrical conductivity, $B(x)$ is the variable magnetic field, $\alpha=\frac{\xi}{(\rho c)_{f}}$ where $\xi$ is the thermal conductivity and $(\rho c)_{f}$ is the heat capacitance of the base fluid, $\tau$ parameter defined by $\tau=\frac{(\rho c)_{p}}{(\rho c)_{f}}, \quad(\rho c)_{p}$ effective heat capacity of

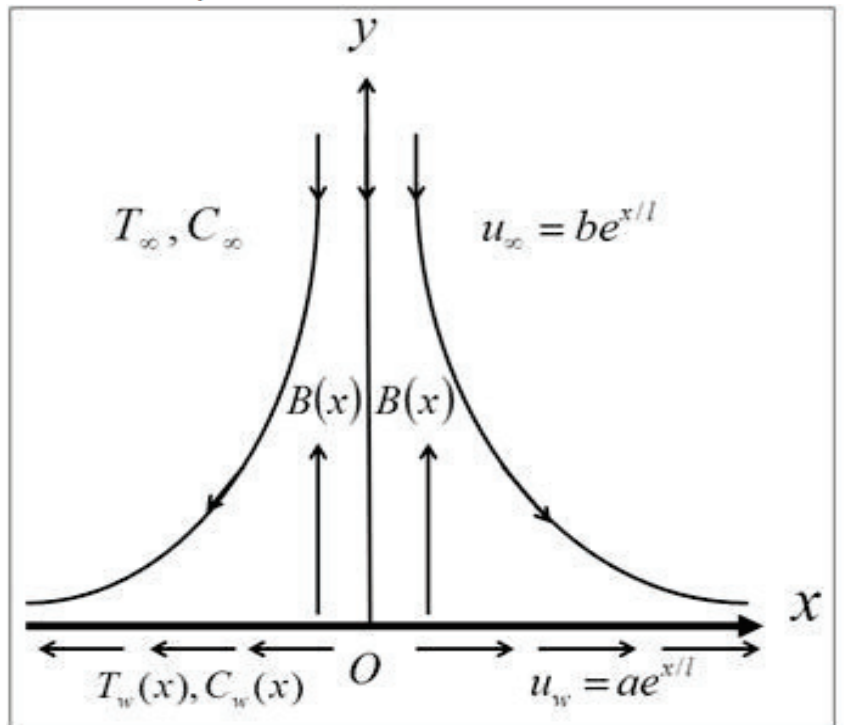

Figure 1. Geometry for the flow under consideration. a nanoparticle, $D_{B}$ is the Brownian diffusion, $q_{r}$ is the radiation flux.

The radiation heat flux is given as

$q_{r}=\frac{-4 \sigma^{*}}{3 k^{*}} \frac{\partial T^{4}}{\partial y}$

where $\sigma^{*}$ is Stefan-Boltzmann constant and $k^{*}$ is mean absorption coefficient. For small temperature differences within the flow, expanding $T^{4}$ about $T_{\infty}$ by Taylor series. This after neglecting the higher order terms takes the following form:

$T^{4}=4 T_{\infty}^{3} T-3 T_{\infty}^{4}$

Substituting (6) in (5), we get

$\frac{\partial q_{r}}{\partial y}=-\frac{16 \sigma^{*} T_{\infty}^{3}}{3 \kappa^{*}(\rho c)_{f}} \frac{\partial^{2} T}{\partial y^{2}}$

Using equation (7) and the energy equation (3) becomes

$u \frac{\partial \mathrm{T}}{\partial \mathrm{x}}+\mathrm{v} \frac{\partial \mathrm{T}}{\partial \mathrm{y}}=\left(\alpha+\frac{16 \sigma^{*} T_{\infty}^{3}}{3 \kappa^{*}(\rho c)_{f}}\right) \nabla^{2} T+\tau\left(D_{B} \nabla \varphi \cdot \nabla T+\right.$

$\left.\frac{D_{T}}{T_{\infty}} \nabla T . \nabla T\right)$

The boundary conditions considered:

$\left\{\begin{array}{c}u=u_{w}(x)=a e^{(x / 2 l)}, \quad v=0, \quad T=T_{w}(x), \\ C=C_{w}(x) \text { at } y=0 \\ u=u_{\infty}(x)=b e^{(x / 2 l)}, v=0, \quad T=T_{\infty}, \\ C \rightarrow C_{\infty} \text { as } y=\infty\end{array}\right\}$

$T_{w}(x)=T_{\infty}+T_{0} e^{(x / 2 l)}, C_{w}(x)=C_{\infty}+C_{0} e^{(x / 2 l)}$ are surface temperature and concentration respectively. Now the nonlinear PDEs are converted into nonlinear ODEs. For that purpose, the stream function $\psi$ is defined by $u=\frac{\partial \psi}{\partial y}, v=-\frac{\partial \psi}{\partial x}$, so that the Eq.(1) will be satisfied identically. For the conversion of the mathematical model (1) (4) into the dimensionless form, the following similarity transformation has been introduced.

$$
\left\{\begin{array}{c}
\eta=y \sqrt{\frac{a}{2 v l}} e^{(x / 2 l)}, \quad \psi=\sqrt{2 v l a} e^{(x / 2 l)} f(\eta), \\
\theta(\eta)=\frac{T-T_{\infty}}{T_{f}-T_{\infty}}, \quad \beta(\eta)=\frac{C-C_{\infty}}{C_{w}-C_{\infty}} .
\end{array}\right\}
$$

The effect of stream function on the remaining three equations, the momentum Eq. (2), the temperature Eq. (3) and concentration Eq. (4) are as

$f^{\prime \prime \prime}+f f^{\prime \prime}-2\left(f^{\prime}\right)^{2}+M\left(A-f^{\prime}\right)+2 A^{2}=0$ 


$$
\begin{aligned}
& \frac{\left(1+\frac{4}{3} N r\right) \theta^{\prime \prime}}{P r}+f \theta^{\prime}-f^{\prime} \theta+N b \theta^{\prime} \beta^{\prime}+N t\left(\theta^{\prime}\right)^{2}=0 \\
& \beta^{\prime \prime}+L e f \beta^{\prime}-L e f^{\prime} \beta+\frac{N t}{N b} \theta^{\prime \prime}-L e \chi \beta=0
\end{aligned}
$$

and the boundary conditions (9) change into the form:

$$
\left.\begin{array}{c}
f(\eta)=0, f^{\prime}(\eta)=1, \theta(\eta)=1, \beta(\eta)=1 \text { at } \eta=0, \\
f^{\prime}(\infty) \rightarrow A, \theta(\infty) \rightarrow 0, \beta(\infty) \rightarrow 0 \text { as } \eta \rightarrow \infty
\end{array}\right\}
$$

Here

$$
\begin{array}{ll}
N r=\frac{-4 T_{\infty}^{3} \sigma^{*}}{3 k^{*} k} & \text { Radiation parameter, } \\
\operatorname{Pr}=\frac{v}{a}, & \text { Prandtl number, } \\
L e=\frac{a}{D_{B}} & \text { Lewis number, } \\
M=\frac{2 l \sigma B_{0}^{2}}{\rho_{f} a} & \text { Velocity ratio parameter, } \\
A=\frac{u_{\infty}}{u_{w}} & \text { Brownian motion parameter, } \\
N b=\frac{\rho_{p} D_{B}\left(C_{w}-C_{\infty}\right)}{\rho_{f} \alpha} & \text { Thermophoresis parameter, and } \\
N t=\frac{\rho_{p} D_{T}\left(T_{w}-T_{\infty}\right)}{\rho_{f} \alpha T_{\infty}} & \\
\chi=\frac{\kappa_{0}}{a} & \text { reaction rate parameter. }
\end{array}
$$

In this problem, the desired physical quantities are the local Nusselt number $N u_{x}$, and reduced Sherwood number $S h_{x}$ and the skin-friction coefficient $C_{f}$. These quantities are defined as

$$
\left.\begin{array}{c}
C_{f x}(0)=C_{f} \sqrt{(2 l / x) R e_{x}}, \\
-\theta^{\prime}(0)=\frac{1}{\left(1+\frac{4}{3} N r\right) \sqrt{(2 l / x) R e_{x}}},-\beta^{\prime}(0)=\frac{S h_{x}}{\sqrt{(2 l / x) R e_{x}}}
\end{array}\right\}
$$

where $R e_{x}=\frac{u_{w}(x) x}{v}$ is the local Reynolds number.

\section{NUMERICAL TECHNIQUE}

The non-linear ordinary differential Equations (11) - (13) with boundary conditions (14) are solved using the $4^{\text {th }}-$ order Adam's-Bashforth predictor and Moulton corrector method along a shooting technique. Equation (11) is solved as an initial value problem assumed $f^{\prime \prime}(0)$. This assumed value is found by Newton's Raphson
Method. This is followed by solving Equations (12) and (13) with assumed values for $\theta(0)$ and $\beta^{\prime}(0)$ correct values are found by shooting method using the end conditions.

Finally, the resulting equations are solved with help of $4^{\text {th }}$ order Adam's-Bashforth predictor and Moulton corrector method along a shooting technique for the step size of $\Delta \eta=0.01$ and $\eta_{\max }=10$ were found to be satisfactory in obtaining sufficient accuracy.

\section{RESULT AND DISCUSSION}

The objective of this section is to analyze the numerical results displayed in the tabular and graphical form. The numerical influence of different parameters for example Brownian motion parameter $\mathrm{Nb}$, thermophoresis parameter $\mathrm{Nt}$, velocity ratio parameter $A$, radiation parameter $N r$, Prandtl number $\operatorname{Pr}$, Lewis number $L e$, chemical reaction $\chi$ and Hartmann number $M$ are given in Tabular form (Tables 1 and 2) and displayed graphically (Figures 2 - 12). Table 1 shows a comparison of obtained results for the reduced Nusselt number $-\theta^{\prime}(0)$ with the results given by $[11,13$ and 14]. These comparisons show an excellent agreement between the obtained numerical results and existing results in the literature.

Table 2 shows the variations of the reduced Nusselt number $-\theta^{\prime}(0)$ and the reduced Sherwood number $-\beta^{\prime}(0)$ for different values of $N b, N t, P r, L e, M, N r, A$ and $\chi$. It is observed form this table that $-\theta^{\prime}(0)$ decreases with the increasing values of $\mathrm{Nb}, \mathrm{Le}, \mathrm{Nr}$ and $\mathrm{A}$. Whereas for increasing values of $\mathrm{Pr}, \mathrm{Nt}, \mathrm{N}$ and $\chi$ is increasing. However, it is found that $-\beta^{\prime}(0)$ decreases for the increasing value of $N r$ whereas it increases for increasing values of $N b, N t, \operatorname{Pr}, L e, M, A$ and $\chi$. Here, it is noted that for the increasing values of $M,-\theta^{\prime}(0)$ and $-\beta^{\prime}(0)$ show a quite opposite effect in both cases of $A<1$ and $A>1$.

Figure 2 shows the effects of $M$ and $A$ on the velocity profiles $f^{\prime}(\eta)$ for fixed values of $N b, N t, \operatorname{Pr}, L e$ when $A<1, A=1$ and $A>1$. This figure shows that $f^{\prime}(\eta)$ decreases for increasing values of $M$ for the case of $A<1$. Here, the decreasing behavior in $f^{\prime}(\eta)$ is justified due to the fact that larger values of $M$ increase the resistive forces on the stretching surface which result in a retardation force to slow down the nanofluid motions However, in the case of $A>1, f^{\prime}(\eta)$ increases for the increasing values of $M$. It is further observed that $f^{\prime}(\eta)$ increases for increasing values of $A$. It is interesting to note that in the case of $A>1$, the momentum boundary layer thickness becomes smaller compared to the case of $A<1$, and causes an inverted boundary layer structure. Moreover, when $A=1, f^{\prime}(\eta)$ coincide with each other and results in a degenerate inviscid flow, where the stretching matches the conditions at infinity. 
Figure 3, represent the effect of Brownian motion parameter on the profile of temperature. So, distribution of Nano particles can be adjusted by adjusting Brownian motion parameter

Figure 4 delineates the influence of $N t$ on the temperature profile. When the effects of thermophoretic increase, the relocation of the nanoparticles relocate from hot part of the surface to the cold ambient fluid and consequently, at the boundary, temperature is increased. This sequels in the thickening of thermal boundary layer.

The influence of radiation parameter on profile of temperature distribution is displayed in Figure 5 . Temperature increases with the increase of thermal radiation parameter $\mathrm{Nr}$. The effect of radiation is to intensify the heat transfer thus radiation should be at its minimum in order to facilitate cooling process.

Figure 6 reveals the influence of $P r$ on dimensionless temperature profile. As expected, increasing $\operatorname{Pr}$ leads to reduction in dimensionless temperature. Based on the definition of $\mathrm{Pr}$ (the ratio of momentum diffusivity to thermal diffusivity), therefore, for large $\operatorname{Pr}$ heat will diffuse more rapidly than the momentum. Consequently, thickness of thermal boundary layer reduces as $\mathrm{Pr}$ increases. It is also noticed that higher values of $\mathrm{Pr}$ reduce the temperature more drastically.

Figure 7 shows that $\theta(\eta)$ increases with increasing values of $M$ when $A<1$ while it decreases in the case of $A>1$. It is noticed that the thermal boundary layer thickness is not much influenced by the larger values of $M$ when $A>1$.

Figure 8 shows the effect of the Brownian motion parameter on the concentration. It is noticed that as the Brownian motion parameter increases, the concentration decreases.

Figure 9 shows the effect of thermal radiation on concentration profile. By increasing thermal radiation, the concentration boundary layer thickness is decreasing.

Figure 10. As Lewis number increases the concentration graph decreases and the concentration boundary layer thickness decreases. This is probably due to the fact that mass transfer rate increases as Lewis number increases. Moreover, the concentration at the surface of a sheet decreases as the values of $L e$ increase.

As it noticed from Figure 11 concentration graph in response to a change in thermophoresis parameter $N t$. The influence of thermophoresis parameter on concentration profile graph is monotonic, i.e. as the values of $N t$ parameter increase, the concentration boundary layer thickness is also increasing.

Figure 12. It is discerned from this figure that the concentration profile diminishes with an increase in the chemical reaction. It is also noticed that nanoparticle concentration and layer thickness shrinkage with the destructive chemical reaction. Considerably, the presence of destructive sources and the presence of the species a cause the chemical reaction which drops the concentration profiles in the concentration boundary layer thickness

TABLE I.

COMPARISON OF $-\theta^{\prime}(0)$ WHEN $N b=N t=L e=A=0$.

\begin{tabular}{|c|c|c|c|c|c|c||}
\hline \hline $\boldsymbol{P r}$ & $\boldsymbol{M}$ & $\boldsymbol{N} \boldsymbol{r}$ & {$[\mathbf{1 3}]$} & Ishak [11] & $\begin{array}{c}\text { Imran ANWAR et al. } \\
{[\mathbf{1 4}]}\end{array}$ & Present Results \\
\hline & & & $-\boldsymbol{\theta}^{\prime}(\mathbf{0})$ & $-\boldsymbol{\theta}^{\prime}(\mathbf{0})$ & $-\boldsymbol{\theta}^{\prime}(\mathbf{0})$ & $-\boldsymbol{\theta}^{\prime}(\mathbf{0})$ \\
\hline 1 & 0 & 0 & 0.9548 & 0.9548 & 0.9548 & 0.9547822 \\
\hline 2 & 0 & 0 & 1.4714 & 1.4714 & 1.4714 & 1.4714600 \\
\hline 3 & 0 & 0 & 1.8691 & 1.8691 & 1.8691 & 1.8690730 \\
\hline 1 & 0 & 1.0 & 0.5315 & 0.5312 & 0.5312 & 0.5317377 \\
\hline 1 & 1.0 & 0 & - & 0.8611 & 0.8611 & 0.8610874 \\
\hline 1 & 1.0 & 1.0 & - & 0.4505 & 0.4505 & 0.4513935 \\
\hline
\end{tabular}

TABLE II.

VAlues of THe Reduced NusSelt Number $-\theta^{\prime}(0)$, The Reduced Sherwood Number $-\beta^{\prime}(0)$ AND SKIN-FRiction COEFFicIENT.

\begin{tabular}{|c|c|c|c|c|c|c|c|c|c|c|}
\hline \hline $\mathbf{N b}$ & $\mathbf{N t}$ & $\mathbf{P r}$ & $\mathbf{L e}$ & $\mathbf{M}$ & $\mathbf{A}$ & $\mathbf{N r}$ & $\boldsymbol{\chi}$ & $-\boldsymbol{\theta}^{\prime}(\mathbf{0})$ & $-\boldsymbol{\beta}^{\prime}(\mathbf{0})$ & $\mathbf{C}_{\mathbf{f x}}(\mathbf{0})$ \\
\hline 0.1 & 0.1 & 1 & 10 & 0.1 & 0.1 & 0.1 & 0.2 & 0.5374883 & 3.4259970 & 1.2856510 \\
\hline 0.5 & 0.1 & 1 & 10 & 0.1 & 0.1 & 0.1 & 0.2 & 0.4683891 & 3.5519440 & 1.2856510 \\
\hline 0.1 & 0.5 & 1 & 10 & 0.1 & 0.1 & 0.1 & 0.2 & 0.5083771 & 2.9029440 & 1.2856510 \\
\hline 0.1 & 0.1 & 10 & 10 & 0.1 & 0.1 & 0.1 & 0.2 & 1.6193550 & 2.9614960 & 1.2856510 \\
\hline 0.1 & 0.1 & 1 & 25 & 0.1 & 0.1 & 0.1 & 0.2 & 0.5358281 & 5.6791770 & 1.2856510 \\
\hline
\end{tabular}




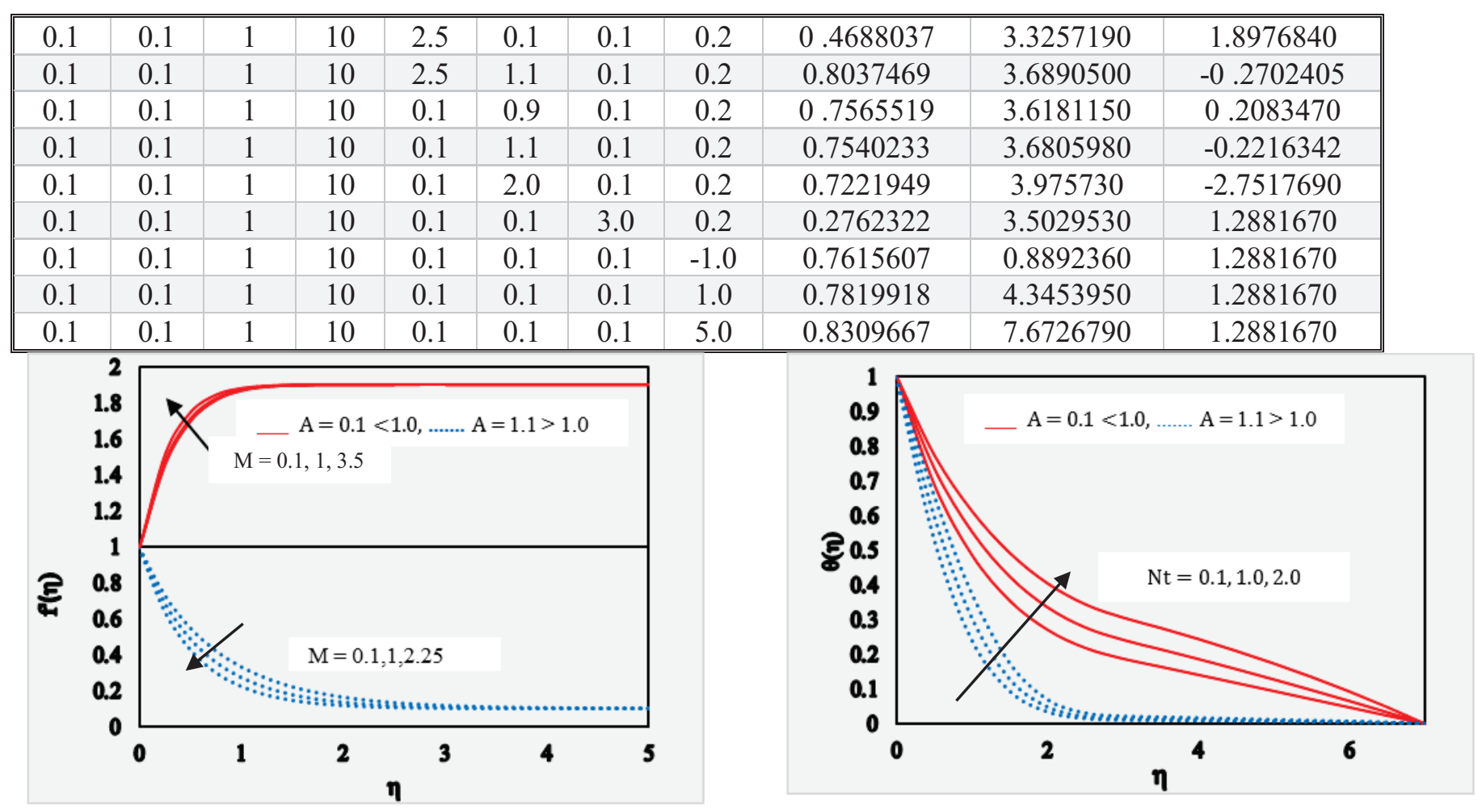

Figure 2. Velocity profiles against $\eta$ for variation values of $M$ when $N t=N b=0.1, P r=L e=2.0, N r=1.0, \chi=0.2$

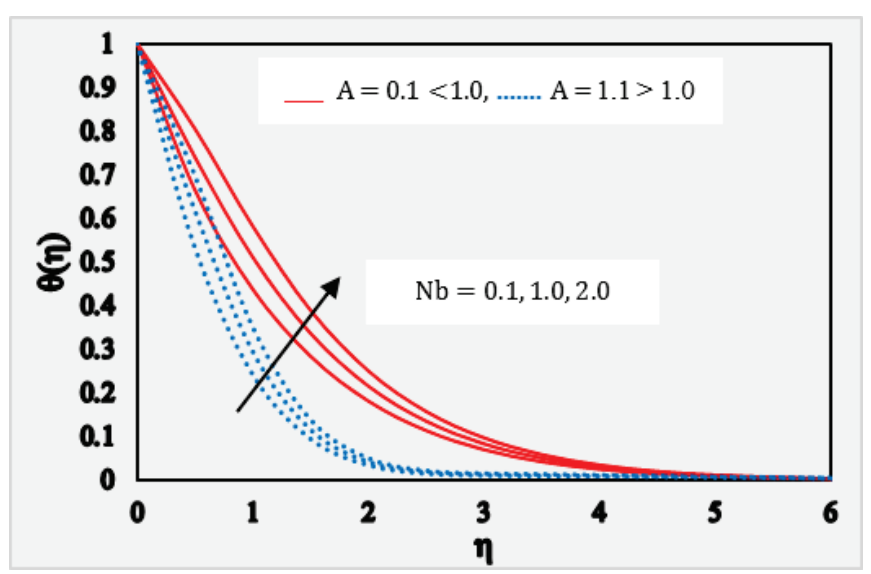

Figure 3. Temperature profiles against $\eta$ for variation values of $N b$, when $N t=M=0.1, P r=L e=2, N r=1, \chi=0.2$
Figure 4. Temperature profiles against $\eta$ for variation values of $N t$, when $N b=M=0.1, P r=L e=2, N r=1, \chi=0.2$

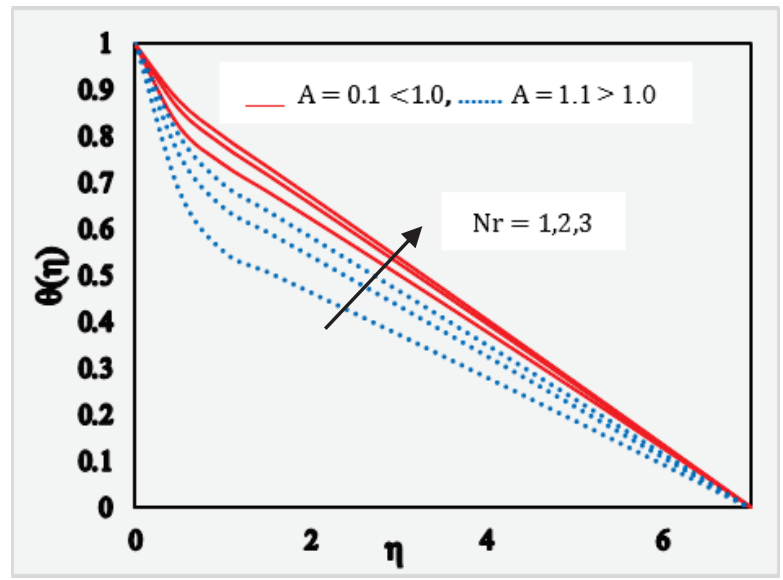

Figure 5. Temperature profiles against $\eta$ for variation values of $N r$ when $M=N t=N b=0.1, P r=L e=2, \chi=0.2$ 


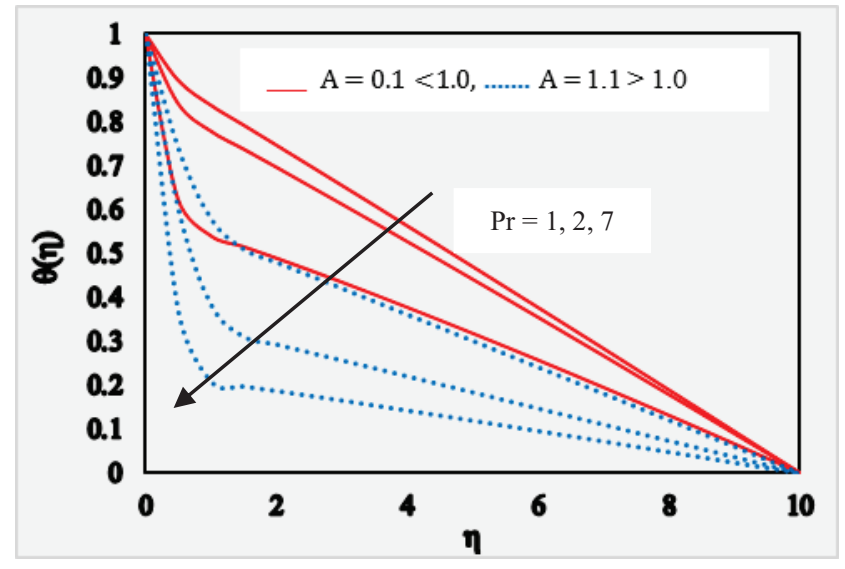

Figure 6. Temperature profiles against $\eta$ for increasing values of $P r$, when $N t=N b=M=0.1, L e=2, N r=1, \chi=0.2$

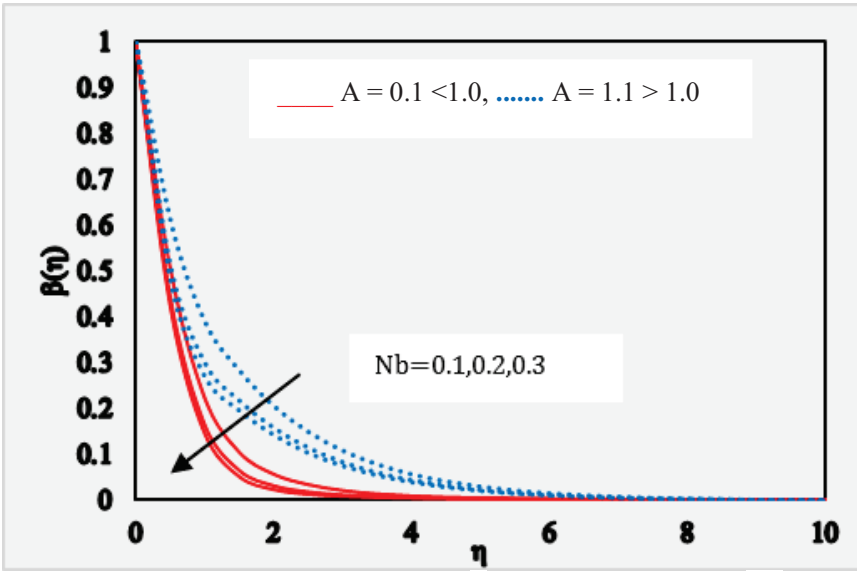

Figure 8. Concentration profiles against $\eta$ for variation values of $N b$ when $N t=M=0.1, P r=L e=2, N r=1, \chi=0.2$

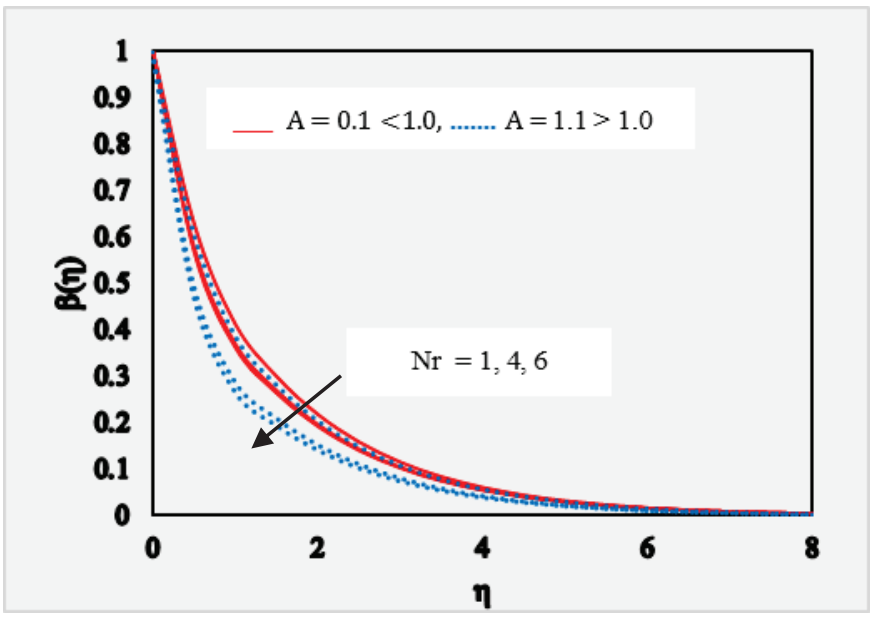

Figure 9. Concentration profiles against $\eta$ for variation values of $N r$, when $N t=N b=M=0.1, P r=L e=2, \chi=0.2$.

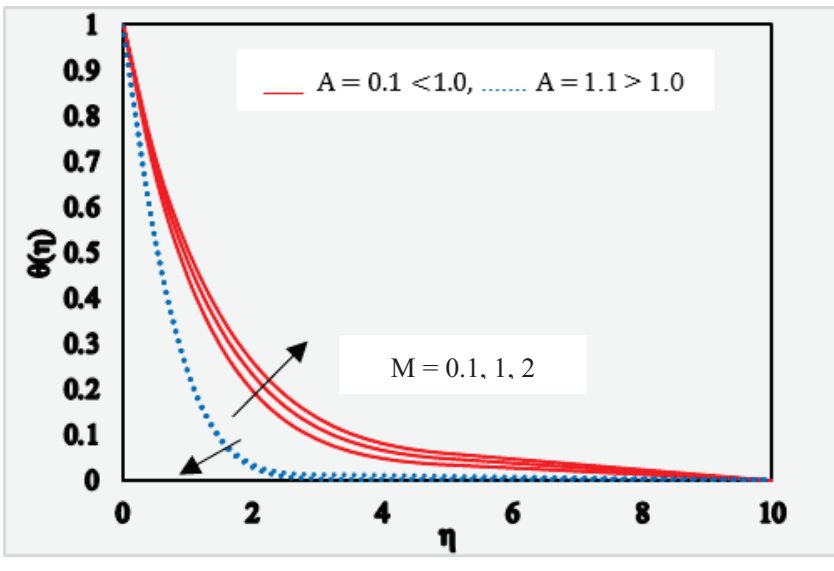

Figure 7. Temperature profiles against $\eta$ for variation values of $M$ when $N b=N b=0.1, P r=L e=2, N r=1, \chi=0.2$

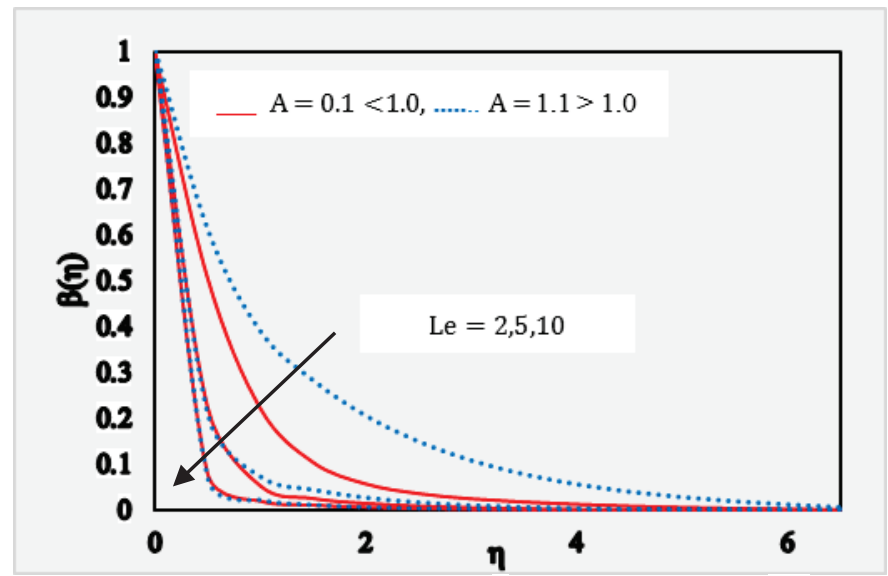

Figure 10. Concentration profiles against $\eta$ for variation values of $L e$ when $N t=N b=M=0.1, P r=2, N r=1, \chi=0.2$

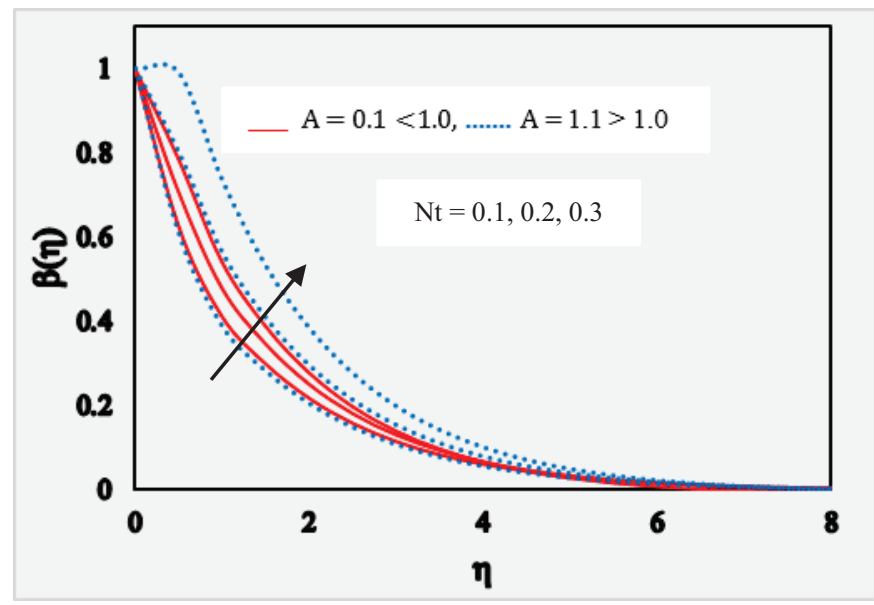

Figure 11. Concentration profiles against $\eta$ for variation values of $N t$, when $N b=M=0.1, P r=L e=2, N r=1, \chi=0.2$. 


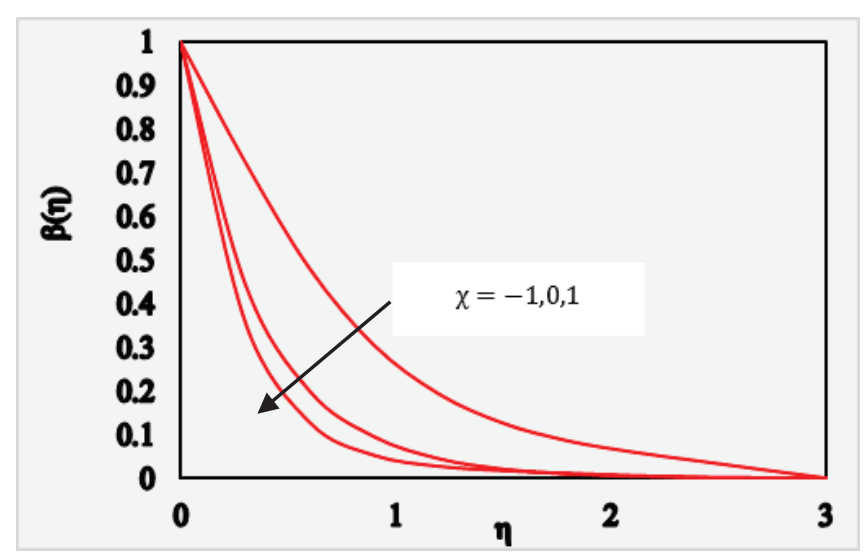

Figure 12. Concentration profiles against $\eta$ for variation values of $\chi$, when $N t=N b=M=0.1, P r=L e=2, N r=1, A=0.1$.

\section{CONCLUSions}

Some of the important findings of this observations are given below:

Dhe velocity profiles conclude with each other if $A=1$ and results in a degenerate inviscid flow where the strecting matches the conditions at the infinity.

$>$ The external stream velocity increases compared to the stretching velocity and the momentum boundary layer thickness shortness for $A>1$, whereas an inverted boundary layer structure is found when $A<1$.

$>$ Increasing values of $M$ has a minimal influence on velocity and temperature profiles for $A>1$.

$>$ Increase in chemical reaction Parameter decrease the concentration profile.

$>$ Increasing Prandtl number decelerates the flow and strongly depresses temperatures throughout the boundary layer regime. while the opposite behavior is seen in case of enhancing the values of Brownian motion parameter, thermophores parameter and radiation parameter.

$>$ We noticed that Concentration is decreased by Brownian motion, Lewis numbers.

\section{NOMENCLATURE}

\begin{tabular}{|l|l|}
\hline$A$ & Velocity ratio parameter \\
\hline$B$ & Transverse Magnetic field $\left(A \cdot \mathrm{m}^{-1}\right)$ \\
\hline$a, b, l$ & Dimensionless needle size \\
\hline$D_{B}$ & Coefficient for Brownian diffusion \\
\hline$D_{T}$ & Coefficient for Thermophoretic diffusion \\
\hline$\xi$ & Thermal conductivity $\left(W \cdot \mathrm{m}^{-1} \cdot K^{-1}\right)$. \\
\hline$L e$ & Lewis number \\
\hline
\end{tabular}

\begin{tabular}{|c|c|}
\hline$M$ & Magnetic parameter \\
\hline $\mathrm{Nb}$ & Brownian motion parameter \\
\hline$N t$ & Thermophoresis parameter \\
\hline $\mathrm{Nu}$ & Nusselt number \\
\hline $\mathrm{Nr}$ & Radiation parameter \\
\hline Nur & Reduced Nusselt number \\
\hline$P r$ & Prandtl number \\
\hline$p$ & Pressure \\
\hline$c_{f}$ & Heat capacity of the fluid \\
\hline$c_{p}$ & Effective heat capacity $\left(\mathrm{J} \cdot \mathrm{kg}^{-1} \mathrm{~K}^{-1}\right)$ \\
\hline$q_{m}$ & wall mass flux \\
\hline$q_{w}$ & surface heat flux \\
\hline$R e_{x}$ & Local Reynolds number \\
\hline Shr & Reduced Sherwood number \\
\hline$S h_{x}$ & Local Sherwood number \\
\hline$T$ & Fluid temperature \\
\hline$T_{w}$ & Fluid Temperature at wall \\
\hline$T_{\infty}$ & Ambient temperature \\
\hline$u, v$ & Velocity vectors \\
\hline$u_{w}$ & Velocity of the stretching surface \\
\hline$x, y$ & Axial and normal coordinates \\
\hline$\alpha$ & Thermal conductivity \\
\hline$\beta$ & Dimensionless nanoparticle volume fraction \\
\hline$\eta$ & Similarity variable \\
\hline$\theta$ & Dimensionless temperature \\
\hline$\chi$ & reaction rate parameter \\
\hline$\rho_{f}$ & Density of Fluid \\
\hline$\rho_{p}$ & Mass density \\
\hline$\sigma$ & Fluid Electrical conductivity \\
\hline$\tau$ & $\tau=(\rho c)_{p} /(\rho c)_{f}$ \\
\hline
\end{tabular}

\section{REFERENCES}

[1] Choi, S.U.S. (1995) Enhancing Thermal Conductivity of Fluids with Nanoparticles. In: Siginer, D.A. and Wang, H.P., Eds. Developments and Applications of Non-Newtonian Flows, ASME, New York, pp 99-105.

[2] Buongiorno, J. (2006) Convective Transport in Nanofluids. ASME Journal of Heat Transfer, 128, pp 240-250.

[3] Cheng, L. (2008) Nanofluid Two Phase Flow and Thermal Physics: A New Research Frontier of Nanotechnology and its Challenges. Journal of Nanoscience and Nanotechnology, 8, pp 3315-3332.

[4] Hu, W. and Buongiorno, J. Nanofluid Coolants for Advanced Nuclear Power Plants. Proceedings of ICAPP'05, Seoul, May 2005, 15-19.

[5] Gupta, P.S. and Gupta, A.S. (1997) Heat and Mass Transfer on a Stretching Sheet with Suction or Blowing. The Canadian Journal of Chemical Engineering, 55, pp 744-746.

[6] Emmanuel, S. and Khan, S.K. (2006) On Heat and Mass Transfer in a Viscoelastic Boundary Layer Flow over an Exponentially Stretching Sheet. International Journal of Thermal Sciences, 45, pp 819-828. 
[7] Subhas Abel, M. and Siddheshwar, P.G. (2007) Heat Transfer in a Viscoelastic Boundary Layer Flow over a Stretching Sheet with Viscous Dissipation and Non-Uniform Heat Source. International Journal of Heat and Mass Transfer, 50, pp 960966.

[8] Nadeem, S., Zaheer, S. and Fang, T.G. (2011) Effects of Thermal Radiation on the Boundary Layer Flow of a Jeffrey Fluid over an Exponentially Stretching Surface. Numerical Algorithms, 57, pp 187-205.

[9] Al-odat, M.Q., Damseh, R.A. and Al-azab, T.A. (2006) Thermal Boundary Layer on an Exponentially Stretching Continuous Surface in the Presence of Magnetic Field Effect. International Journal of Applied Mechanics and Engineering, 11, pp 289-299.

[10] Chand, G. and Jat, R.N. (2014) Flow and Heat Transfer over an Unsteady Stretching Surface in a Porous Medium.Thermal Energy and Power Engineering, 3, pp 266-272.

[11] Ishak. MHD boundary layer flow due to an exponentially stretching sheet with radiation effect. Sains Malays. 2011; 40, pp 391-395

[12] Sudhakar, K., Srinivas Raju, R. and Rangamma, M. (2013) Hall effect on an unsteady MHD flow past along a porous flat plate with thermal diffusion, diffusion thermo and chemical reaction, Journal of Physical and Mathematical Sciences,2013; 4, pp 370 -395 .

[13] B Bidin and R Nazar. Numerical solution of the boundary layer flow over an exponentially stretching sheet with thermalradiation. Eur. J. Sci. Res. 2009; 33, pp 710-7.

[14] Imran ANWAR, Sharidan SHAFIE and Mohd Zuki SALLEH (2014) Radiation Effect on MHD Stagnation-Point Nanofluid over an Exponentially Stretching Sheet, Walailak J Sci \& Tech 2014;11(7): pp569591. 